Methods Triplicate confluent primary human microvascular endothelial cells (Promocell $\mathrm{GmbH}$ ) were cultured in the presence and absence of TGF-beta stimuli relevant to pulmonary arteriovenous malformations and hereditary haemorrhagic telangiectasia (HHT). Following rRNA-depletion of total RNA, seven libraries were prepared using Illumina reagents, and $8 \mathrm{pM}$ of each library used for cluster generation and sequencing on Genome Analyser II. Algorithms for aligning reads to NCBI36/ hg18 and GRCh37/hg19 included Bowtie, TopHat and Seqmap. Validations were performed using quantitative rt-PCR.

Results More than 2 Gigabases of sequence was generated. Transcriptome-wide profiles were similar between libraries, with sixteen types of RNA species detected including 146 micro (mi) RNA families (47 broadly conserved), and 10,749 proteinencoding mRNAs representing $\sim 5.5 \%$ of mapped reads. Alignments to endothelial mRNAs/miRNAs were substantially higher than to gene loci for non-endothelial mRNAs/miRNAs. mRNA exon alignments demonstrated sharp exon boundary delineation, but replicate alignments to non-repetitive intronic regions involved in multi-exon deletions in HHT patients. There was an inverse relationship between alignments depths and qt-PCT cycle thresholds $(\mathrm{Ct})$, where single alignments were detectable, and $\mathrm{Ct}$ values of 20 generated by $0.02 \mathrm{nM}$ spiked RNA. Across all experiments in replicate donor/treatment RNAs, for a panel of single open reading frame miRNA genes, RNASeq alignments (gene strand read counts normalised to the total number of valid reads and exon/locus size) explained $72 \%$ of the variance of qtPCR cycle threshold $(p<0.0001)$. Dynamic whole transcriptome profiling is in progress.

Conclusions These novel directional next generation RNA sequencing methods provide new insights for mutational mechanisms, and a systems approach to dissection of regulatory and target RNA networks relevant to human disease.

\section{P16 A SYSTEMATIC CHARACTERISATION OF INFLAMMATION IN CHRONIC THROMBOEMBOLIC PULMONARY HYPERTENSION}

C Hadinnapola, M Southwood, D Jenkins, K Sheares, M Toshner, J Pepke-Zaba. Papworth Hospital NHS Foundation Trust, Cambridge, UK

\subsection{6/thoraxjnl-2014-206260.166}

Introduction The pathogenesis of chronic thromboembolic pulmonary hypertension (CTEPH) is poorly understood. Idiopathic pulmonary arterial hypertension (IPAH) is associated with systemic and localised inflammation. Distal vasculopathy is seen in IPAH and CTEPH (2 compartment model). Inflammation has also been implicated in CTEPH pathogenesis. We undertook a systematic assessment of inflammatory markers and immune cells to determine the role of inflammation in CTEPH.

Methods We examined the distal tails of 26 pulmonary endarterectomy (PEA) specimens and explanted lungs (5 CTEPH, 11 IPAH). Formalin fixed samples were immunostained with antihuman CD45 (inflammatory cell), CD79a (B cell), CD68 (macrophage) or CD3 (T cell) antibodies. Cell counts were normalised to the area surrounding vessels bounded by airspace structures for perivascular cell counts; to vessel area for media cell counts and per high powered field for lung parenchymal cell counts. Serum was collected from 61 patients pre and 6 months post PEA. Cytokines were measured using a multiplex array $(\mathrm{pg} / \mathrm{ml}$; mean $\pm \mathrm{SD})$.

Results Table 1.

$\mathrm{CD}^{+}$cells were less abundant in the media of small vessels (mean $0.03 \pm 0.01 \mathrm{~mm}^{2}$ ) in CTEPH vs. IPAH $(\mathrm{p}=0.02)$.
Perivascular and parenchymal cell counts showed non-significant reductions of $\mathrm{CD}^{+}$cells in CTPEH $(\mathrm{p}=0.19$ and 0.08 respectively). More $\mathrm{CD} 68^{+}$cells were seen in the parenchyma in CTEPH $(\mathrm{p}=0.03)$. Few $\mathrm{CD} 20^{+}$cells were seen in parenchyma with no difference.

Neovascularisation correlated to $\mathrm{CD}_{4} 5^{+}$cells in PEA specimens when normalised to specimen area $(\mathrm{r}=0.4, \mathrm{p}=0.01)$.

Conclusions In CTEPH, most serum cytokines were not elevated sufficiently to suggest systemic inflammation is important in pathogenesis. The change in IL10 and TNF $\alpha$ are possibly due to improvement in cardiac function post PEA as described previously. The relative lack of $\mathrm{CD}^{+} \mathrm{T}$ cells in the media of small arteries in CTEPH suggests that localised inflammation is also less important. The significance of $\mathrm{CD}^{+} 8^{+}$cells in CTEPH lung parenchyma needs further assessment.

The correlation of $\mathrm{CD} 45^{+}$cells and neovascularisation in PEA specimens may suggest an association between inflammation and neovascularisation.

Inflammatory signals maybe related to thrombus remodelling and cardiac dysfunction rather than to CTEPH pathogenesis. Isolating these processes is required to expand on this descriptive study.

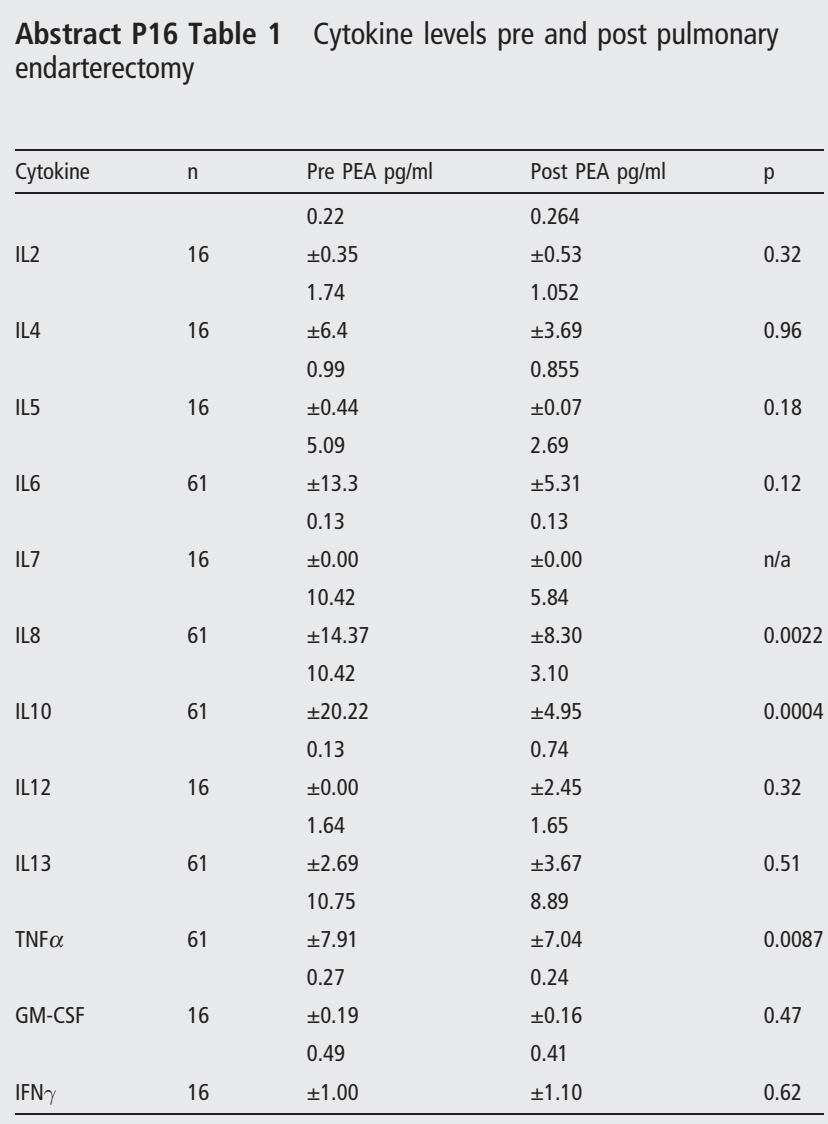

Data presented as mean \pm SD. $p$ - Wilcoxon rank-sum test. IL - interleukin; TNF $\alpha$ tumour necrosis factor $\alpha$; GM-CSF - granulocyte-macrophage colony-stimulating factor; IFN $\gamma$ - interferon

P17 MOLECULAR COMPLEXITIES IDENTIFIED THROUGH
TARGETED GENOMIC SEQUENCING OF THE HHT3 LOCUS ON CHROMOSOME 5

CL Shovlin, IG Mollet, A Giess, E Thomas, MD Jones, L Game, FS Govani. Imperial College, London, UK

10.1136/thoraxjnl-2014-206260.167 